\title{
$\begin{array}{r}\text { WAGENINGEN } \\ \text { UNIVERSITY \& RESEARCH } \\ \hline\end{array}$
}

\section{Multimodal data to design visual learning analytics for understanding regulation of learning}

Noroozi, O., Alikhani, I., Järvelä, S., Kirschner, P. A., Juuso, I., \& Seppänen, $\mathrm{T}$.

This is a "Post-Print" accepted manuscript, which has been Published in "Computers in Human Behavior"

This version is distributed under a non-commercial no derivatives Creative Commons (c) $(1 \otimes \Theta$ reproduction in any medium, provided the original work is properly cited and not used for commercial purposes. Further, the restriction applies that if you remix, transform, or build upon the material, you may not distribute the modified material.

Please cite this publication as follows:

Noroozi, O., Alikhani, I., Järvelä, S., Kirschner, P. A., Juuso, I., \& Seppänen, T. (2019). Multimodal data to design visual learning analytics for understanding regulation of learning. Computers in Human Behavior, 100, 298-304.

https://doi.org/10.1016/j.chb.2018.12.019

You can download the published version at:

https://doi.org/10.1016/j.chb.2018.12.019 


\title{
Multimodal Data to Design Visual Learning Analytics for Understanding Regulation of
}

\section{Learning}

Omid Noroozi* a,b, Iman Alikhani ${ }^{\mathrm{a}}$, Sanna Järveläa, Paul A. Kirschner ${ }^{\mathrm{a}, \mathrm{c}}$, Tapio Seppänen ${ }^{\mathrm{a}}$, \& Ilkka Juuso $^{\mathrm{a}}$

\author{
${ }^{\text {a }}$ University of Oulu, Finland \\ ${ }^{\mathrm{b}}$ Wageningen University, the Netherlands \\ ${ }^{\mathrm{c}}$ Open University of the Netherlands
}

* Authors' note:

Correspondence concerning this article should be addressed to Omid Noroozi, Education and Learning Sciences Group, Wageningen University, P.O. Box 8130, NL 6700 EW, Wageningen, the Netherlands. E-mail: omid.noroozi@wur.nl; omid_noruzi@yahoo.com

Acknowledgements

This study was supported by the Finnish Academy grant 275440 . 


\title{
Multimodal Data to Design Visual Learning Analytics for Understanding Regulation of Learning
}

\begin{abstract}
The increased interest in multimodal data collection in the learning sciences demands for new and powerful methodological and analytical techniques and technologies. It is especially challenging for learning scientists to handle, analyse, and interpret complex and often invisible multimodal data when investigating regulation of learning in collaborative settings as this data can be cognitive, social and/or emotional in nature, much of which is covert in nature. The aim of this paper is to present ways to simplify the analysis and use of rich multimodal data by learning scientists. This is done by making primarily invisible regulation processes and their accompanying social and contextual reactions visible, measurable, and ultimately interpretable. To facilitate data visualisation and processing with respect to the regulation of learning, a Graphical User Interface (GUI) known as SLAM-KIT has been designed. SLAM-KIT reveals principal features of complex learning environments by allowing users to travel through the learners' data and its statistical characteristics. This kit has practical implications as it simplifies complex information and data while making them available through visualisation and analysis to the researchers. Our short-term goal is to simplify this tool for the teachers and learners. Key words: Collaborative learning, Learning, Learning analytics, Regulation of learning, Multimodality
\end{abstract}




\section{Multimodal Data to Design Visual Learning Analytics for Understanding Regulation of Learning}

\section{Introduction}

Working and learning in the $21^{\text {st }}$ century require knowledge building and sharing in teams (Noroozi et al., $2012 \& 2018$ ). Learning is no longer seen as being only cognitive, but is now seen as a complex process that is also social and emotional in nature (Järvelä et al., 2016; Malmberg et al., 2017; Zimmerman \& Schunk, 2011). To better understand this complexity of learning in all of its nuances and intricacies, learning scientists look for ways to gather multimodal data from different channels which may help them reveal the complex interaction of the invisible mental and metacognitive learning process (Malmberg et al., 2017).

Self-Regulated Learning (SRL) theory explains the core mechanisms of interaction of cognition, motivation and emotion in a learning process (Zimmerman \& Schunk, 2011) which is seen as a cyclical complex metacognitive and social process that involves adapting thought, motivation, emotion, and behaviour (Winne \& Hadwin, 1998). Since learning situations are increasingly social and interactive, SRL theory has increasingly been considered in both individual (see Basol \& Balgalmis, 2016) as well as social and collaborative contexts (Järvelä \& Hadwin, 2013; Wise \& Hsiao, in press). Since many learning activities take place at the group level in modern classrooms, the focus of this paper is on SRL in collaborative settings. Three types of regulated learning have been conceptualized as central processes in interactive and collaborative learning contexts (Järvelä \& Hadwin, 2013). These are: SRL which is primarily individual; Co-Regulated Learning (CoRL) which deals with the interaction between pairs of learners where one learner prompts or scaffolds the learning activities of the other, and Socially Shared Regulation of Learning, (SSRL) which occurs in groups where the team as a whole regulates the learning activities of and within the team. Scientific literature suggests that proper planning and strategic adaptation of one's learning to challenges during the learning process requires being able to strategically regulate oneself (i.e., SRL), each other in a dyad or group (i.e., CoRL), and the individuals in a group together (i.e., SSRL) (Hadwin et al., 2017). When investigating collaborative interactions, learning peers generate mountains of data on what is attended to and studied, in what order this occurs, how much time was spent on what, at what times certain actions occurred, at which places in the study environment, et cetera. In collaborative settings where learners learn together, the amount of data generated increases exponentially (Molenaar \& Chiu, 2014). Learning Analytics (LA) can be used to deal with such large amounts of data and make sense of it for both learner and teacher (see Nistor \& Hernández-Garcíac, 2018; Viberg et al., 2018). LA is the data-driven analysis of learning activities and environments involving capturing, tracking, 
collecting, analysing, and reporting of data about learners and their contexts to understand learning and the learning environment and, ultimately, optimise learning (Gašević et al., 2015; Verbert et al., 2013). As such, LA can be used to analyse and visualise learning patterns and provide learners and teachers with information to optimise the cognitive, social, and emotional components of individual, peer, and collaborative learning (Roll \& Winne, 2015). LA, thus, can inform learners and teachers preferably in real-time - of what individuals actually are doing and what is being achieved so as to trigger and sustain learning and its progress (Greller \& Drachsler, 2012). This approach can also be expanded to large scale collaborative and collective learning situations such as learning communities (Mikroyannidis et al., 2013; Zhang et al., in press).

Learning in teams is increasingly occurring with the aid of technology and artefacts available in the learning environment (Miller \& Hadwin, 2015). There has been much progress in the development of interesting and powerful technologies, with new data-capturing devices such as eye-tracking glasses (Jarodzka et al., 2017), wearable physical and/or biophysical sensors, and high speed recording devices to capture facial micro-gestures (Li et al., 2017). The combination of such technologies allows researchers to go beyond simple ontologically flat data on observable or selfreported behaviours (i.e., data that gives an event-focused view of learning phenomena that merely provide descriptive accounts of events) to multimodal data sets that simultaneously trace a range of cognitive and non-cognitive processes, including nearly invisible micro-level environmental interactions and invisible responses of the body and brain (Reimann et al., 2014). Such multimodal data can provide new supplementary and complementary methods for capturing important phases of regulated learning as they occur in challenging learning situations (Harley et al., 2015).

Although, there has been significant theoretical and conceptual progress with respect to regulation in learning, there has been little progress in developing methods to make the primarily invisible mental regulation processes and their accompanying social and contextual reactions visible and thus measurable and ultimately interpretable. This is important since there is a potential to visualise, understand, make sense of, and ultimately use such data in various learning situations to inform learners and their collaborative partners about their own and other's learning regulation processes on the one hand and the teacher or instructor on the other. Capturing and then combining multimodal data can provide researchers, teachers and students with fun-damentally new objective and subjective ways to view and deal with the critical phases of SRL, CoRL, and SSRL processes, find evidence of critical moments of success or failure during learning, and then act upon this information to make the learning more effective and efficient (and possibly even more enjoyable by helping to mediate or avoid cognitive, social, or emotional conflicts). However, it is challenging to handle, analyse, and 
interpret this complex multimodal data when investigating regulation of learning in collaborative settings. Each of the different data sources and techniques is interpretable and usable only in its own right (e.g., Electro Dermal Activity (EDA) can be used to signal stress, heart rate for exertion, et cetera) but the challenge is to combine different measures to be able to interpret them in terms of their relation to and effects on learning (e.g., through the use of video data understanding what the cause was of a change in EDA or heart rate).

This paper presents an integrated analysis tool which is known as SLAM-KIT. SLAM is a project funded by the Finnish Academy of Science (project number 275440) which stands for Strategic regulation of learning through Learning Analytics and Mobile clouds for individual and collaborative learning success. SLAM harnesses advanced technologies to enhance strategic regu-lation of individual and collaborative learning.

The aim of this paper is to simplify the use and analysis of rich multimodal data recorded from collaborative learning situations using SLAM-KIT. We begin by de-scribing SRL, SSRL, and CoRL as the methodological background for the tool. We then explain how and why various multimodal data should be combined followed by a short description on how these multimodal data can be visualised in the SLAM-KIT. Next, we pay specific attention to the system architecture of the SLAMKIT and its functionality after which we present findings from using the tool to analyse a set of recorded learning situations as realworld case examples. We conclude by reflecting on the practical implications of the tool for the learning sciences.

\section{Understanding complex multimodal data for regulation in learning}

Multimodal data typically originates from various data channels which may be subjective or objective. This data enables researchers and educators to capture various aspects and phases of learning regulation as they occur in challenging learning situations (Harley et al., 2015). Until now, most approaches for regulated learning measurement have relied on traditional subjective measures such as self-reported data of learners' own intentions, beliefs, and perceptions on their learning experiences of how they would/did enact during learning processes (Zimmerman, 2008). However, these subjective measures are inadequate for coherently and reliably capturing the complexity of SRL, CoRL, and SSRL (Bannert et al., 2014). Although such self-reported data might reveal learners' stated intentions to enact and their beliefs about themselves as learners, they often do not match with what actually happened during learning processes (Winne, 2004; Zimmerman, 2008). Learning scientists suggest that such data can be complemented with other, more objective and objectively measured data such as log data, video observations, physiological, and biophysiological measures to adequately inform the deployment of the regulatory process especially in social learning contexts. 
Physiological reactions of learners, for example, can be seen as informative data that can be used for signaling and measuring regulation mechanisms during the learning process (Pijeira-Díaz et al., 2016). Such data can provide information about various aspects of learners' actual behavioural, physiological, and mental processes that coincide with events such as study choices, confusion, and changes in effort or attention in a learning situation that are almost impossible to capture otherwise (Henriques et al., 2013; Winne, 2010). For instance, cardiovascular data provides information on arousal and heart rate variation. Such cardiovascular data have been found to be related to experienced cognitive load (e.g., Cranford et al., 2014; Haapalainen et al., 2010).

Another important physiological indicator known as Electro Dermal Activity (EDA; also known as galvanic skin response) refers to the electrical conductivity properties of the learner's skin that can provide information on activities and arousal of learners' sympathetic nervous system (Garbarino et al., 2014). Skin Conductance Responses (SCRs) is a phasic measure of EDA elicited by stimuli that reflects rapid changes in the EDA signal. Tonic measures of EDA are called Skin Conductance Level (SCL) and Non-Specific Skin Conductance Responses (NSSCRs) elicited by unfolding events that reflect long-term changes. Such SCL and NSSCRs measures provide information on cognitive appraisals of learners related to their goal relevance (e.g., Kreibig et al., 2012) and also the perceived task difficulty leading to emotions (e.g., Pecchinenda \& Smith, 1996; Tomaka et al., 1993). There are other physiological modalities such as eye-movement, facial gesture, and micro-gesture recognition data that are related to the learners' cognitive demands indicating the extent to which they are bored, confused, stressed, and so on during the learning process (D’Mello, 2013; Fairclough et al., 2005).

In modalities, physiological data obtained through direct and objective measurement such as EDA (Chanel \& Mühl, 2015; Pecchinenda \& Smith, 1996), with eye-movement, and facial recognition data (D’Mello, 2013; Fairclough et al., 2005) can be complemented with observational data such as audioand video of learning situations (Malmberg et al., 2017) and traditional subjective measures such as self-report (Zimmerman, 2008). Combining these different types and modalities of data can provide researchers with a set of indicators for reflecting on regulation mechanisms during learning process. Whilst physiological measures such as EDA can be used to trace skin reactivity changes of learners in challenging moments (i.e., emotional arousal; Chanel \& Mühl, 2015; Pecchinenda \& Smith, 1996), video data can be used to reveal sequential and temporal dynamics of learners' regulated learning (i.e., planning, enacting strategies, reflection, adaptation) which can help explain the observed physiological data. Such data on temporal and cyclical processes of regulation make it possible to see how previous small-scale situated adaptations and regulation of situated challenges contribute to large-scale adaptation during collaborative learning (Hadwin et al., 2017). Furthermore, such data 
help reveal patterns of activation of regulatory processes (i.e., planning, goal setting, enacting strategies, motivation) to see how possible sequences of regulated learning contribute to learning progress (Malmberg et al., 2017). These fine-grained objective data and an examination of the relations among different data sources and variables can help elucidate hidden physiological reactions that are practically invisible and thus nearly impossible to capture.

In the next section, we explain how the combination of multimodal data can make the primarily invisible learner's cognitive and non-cognitive regulation processes and their accompanying social and contextual reactions visible. Such visualisation can signal and inform learners and their collaborative partners about their own and other's learning regulation processes.

\section{Multimodal data combination and visualisation: A research case example}

The use of multimodal data in general and learning regulation in particular is emerging and progressing quickly in the learning sciences (Antonietti et al., 2014; Blikstein et al., 2016; Harley et al., 2015; Worsley \& Blikstein, 2015). Single physiological markers can only capture affective states of learners in complex learning situations (D'Mello et al., 2017) without accompanying context to aid in/allow for interpretation. This is striking since learning regulation is not simply cognitive or affective but is multifaceted requiring learners to be able to take control of their motivation, emotion, affect, behavior, and cognition influencing each other. Such multimodal data collection methods can advance the field of learning sciences by revealing learning regulation processes that are impossible to achieve with conventional and traditional educational psychology methods (Azevedo, 2015).

In the following section, we demonstrate how multimodal data can be combined and visualised using a real set of captured data. We present a case example from our study, which focused on investigating socially shared regulation in advanced high school physics collaborative learning tasks. The study aimed to integrate time-stamped video-data and corresponding physiological data to contextualise physiological reactions to specific learning situations. This was done to find new methods for identifying individual students' physiological markers and signals that may or may not be relevant for identifying socially shared regulation of learning in groups.

The SLAM project aims to understand and make visible learners' invisible complex, cognitive, motivational, and emotional learning processes to help them regulate those processes and thus learn more effectively, efficiently, and hopefully enjoyably. This would enhance learning by making available the unprecedented amount of data that learners generate during learning process, interacting with teachers and peers, and creating new shared knowledge. Making information visible implies that even though learning regulation is considered a psychological phenomenon (Winne, 2017), it also 
has physiological indicators such as stress, excitement, enthusiasm, or emotional dynamics in groups (Mønster et al., 2016). Our multimodal data collection (including 101 hours of video data, 266,215,652 sensor data points, and 236,122 log data entries) has resulted in a rich dataset about students' learning processes in collaborative tasks. The challenge has been to make the large amount of complex multimodal data readily accessible in a unified and visual manner for learning scientists to study the phenomena underlying the learning processes during collaboration. Making sense of such complex multimodal data can also be useful in educational data mining and learning analytics (Roll \& Winne, 2015) and ultimately to develop adaptive regulation support (i.e., support that phases out as the learner masters it) for individuals and groups and make them visible and, thus, supportable for researchers and teachers.

\section{System architecture of the SLAM-KIT}

The SLAM-KIT is an integrated analysis tool for the learning sciences that simplifies the use and analysis of rich multimodal data recorded from collaborative learning situations. It enables users to dive into recorded interaction situations not only via the video and audio of the situation, but also with a wealth of physiological information captured during the situation using unobtrusive sensors and cameras. The tool merges the diverse data sources and provides a unified navigable view of the entire interaction situation. The video and audio are aligned with sensor data, which is then presented as color-coded plots on the same timeline. The goal is to provide learning scientists with easy access to all of the physiological information relevant in the SRL, SSRL and CoRL approaches to analyse the learning situations.

SLAM-KIT is developed in MATLAB 2017a using the GUI Development Environment (GUIDE) model. The tool uses MATLAB's image processing and statistics and machine learning toolboxes in the handling of the physiological signals and an ActiveX-enabled media player module for all playback functionality. Like most GUIs, SLAM-KIT consists of a layout, which pops up for the user (known as front-end), and an implementation code environment (known as back-end) containing the call-backs of the control objects, including buttons panels, sliders axes and axes algorithms. The life cycle of the SLAM-KIT is depicted in Fig 1.

The current version of the SLAM-KIT is a standalone piece of software. The tool, therefore, requires installation on a PC and the changing of a small number of practical settings (e.g., where to find the data). The tool will be developed further by the research group. Once developed, these configurations will be accessible to the users through the digital website of the SLAM project. 
The tool is customized for our data protocol and for the participants' setting. There is a predefined structure for the annotations and bio-signals in a comma separated format (i.e., csv) given the master clock set based on the video data and other data stream adjusted based on the video clock. The generalisation of the SLAM-KIT (i.e., to execute for different number of participants, to perform more signal processing methods, and to provide an interface for different settings with similar context) is possible and is in the future work plan of the researchers. Figure 1 illustrates the overall data architecture of the SLAM-KIT environment.

\section{Insert Figure 1 about here.}

When first opening up a particular session in the tool, the user is asked to insert the group number they wish to analyse from the groups recorded during the session. The software then loads the corresponding data available for the session, including the video, audio, physiological data and any manually entered labels or annotations in the stack memory (see Fig.1). The tool then displays an overview of the session where the synchronized signals are plotted a) separately for each subject (see Fig. 2) and b) jointly in one master view for all subjects together (see Fig. 3). The former view (Fig. 2) is practical for following any subject separately while the latter view (Fig. 3) is intended to facilitate discovery of influence between subjects. The software and user interaction is by the designed GUI, as sketched in Fig. 1, and the back-end script is linked with the memory, GUI, and also to the storage (to restart and switch the study group).

The top array of Fig 2 shows the physiological signals synchronized with the video for each subject. In the middle, an annotation axis plot shows the annotation labels with time, duration and type. Additionally, more information about the labels is given in the annotation table (lower left-hand corner) and, above this table, quantitative information is given on the analysis of the signals. The video playback panel shows the recorded video with standard playback controls. The Controllers panel (lower right-hand corner) contains additional options for an advanced user. Fig. 3 depicts the master view of a collaborative learning session that combines the plots for each subject by signal type. Top four plots in the Fig. 2 correspond to the temperature, EDA, BVP and HR biosignals, while the fifth plot shows the annotations assigned to each point in time. Similar to the sensor data, also the annotated labels are visualised along the time axis, as well as in table-form. Clicking on any plot affects the playback of the video, thereby making the process of browsing the data straight-forward. Insert Figures $2 \& 3$ about here.

SLAM-KIT also enables users to choose and execute simple analysis algorithms to identify and measure phenomena relevant in their investigation. These include algorithms for Multidimensional 
Recurrence Quantification Analysis (MdRQA) (Wallot, Roepstorff, \& Mønster, 2016), slope coding (Leite, Henriques, Martinho, \& Paiva, 2013) and Pearson correlation coefficient among the students' signals. Depending on the user's research question, any externally prepared piece of analysis and code could be merged into the software. This exemplified set of processing methods offers the physiological coupling quantification of biosignals. MdRQA computes a number of metrics from the recurrence plot (Fig. 4) that, for example, measure the level of synchrony between the biosignals. Pearson correlation coefficient is also well-recognized method to compute the correlation between pairs of signals. The slope coding model quantizes the slope fluctuation of EDA signals to a limited number of bins and offers a histogram of the signal variation. These algorithms can be run on the entire learning session or a selected shorter segment of the session by controlling the time axes.

We use a software architecture that can be easily extended with new analysis functions that are hypothesized to be relevant for representing the regulation of learning or any useful interaction among the group. MdRQA is an extension of recurrence quantification analysis (a nonlinear data analysis method) which derives metrics from recurrence plots. A recurrence plot visualizes the periodic nature of physiological signals (in our case) with respect to the time. The idea of MdRQA is to find the synchrony among the students from physiological signals. Samples of resulting analysis plots are illustrated in Fig. 4, which shows MdRQA and slope coding analysis windows. As can be seen in Fig. 4, in the left window, a recurrence plot of EDA and heart rate signals is depicted along with quantifications displayed above the sub-figures. In the right window the slope coding method and its histo-gram is depicted for each subject's EDA signal.

\section{Insert Figure 4 about here.}

\section{Case example of SRL data visualisation}

SLAM-KIT facilitates the coordinated inspection of biosignal variation with respect to predefined annotations. One can investigate the reflection of cognition, motivation, and emotion interactions in a learning process in the recorded synchronized biosignals and its analysis.

In this section, to present further details and capabilities of the kit, a number of observations from the group exam sessions is provided. Fig. 5 is a snapshot taken of the data for the three students from group two at the beginning of the exam. The annotation includes a set of cognition interaction labels for events sustained for at least one minute in length. At the same time, the heart rate, temperature and EDA signals show high level of correlation between ID48 and ID50. The EDA signals of these two students, shows a phasic arousal around seven minutes into the session. Similarly, their heart rate and temperature show a local maxima and similar morphology in this period. There seems to be a 
physiological coordination between these two students at the given time period while a set of cognition interactions are observed.

\section{Insert Figure 5 about here.}

Similarly, there is a series of monitoring cognition events between 9.5 and 11.0 minutes into the session, as depicted in Fig. 6. In this case, there is a contradiction between the heart rate of ID49 and ID50, while their temperature is gradually rising with the same space.

\section{Insert Figure 6 about here.}

A series of monitoring cognition events followed by a monitoring behaviour event is in the selected segment of group one data (see Fig. 7). There seems to be a reasonably high level of contradiction with an apparent delay between the temperature and heart rate of ID46 and ID73. However, the EDA and heart rate of ID47 and ID73 shows a correlation in their fluctuation.

\section{Insert Figure 7 about here.}

\section{Implications and conclusions}

The SLAM project has been working to find new ways to explore socially shared regulation in collaboration, since earlier existing methods have not been powerful to describe the social and metacognitive aspects of cognition, motivation and emotion and their regulation. The major claim is that understanding regulatory processes in collaboration requires triangulation of multimodal data. Multimodal and multichannel data collection is a new opportunity to understand temporally unfolding SRL processes, especially its complex process of self-regulation involving cognition, motivation, and emotion as a strategic regulatory activity. The focus of this study is on the notion of regulation of learning referring to the ways learners systematically activate and sustain their cognitions, motivations, behaviors, and affect towards attainment of their learning goals. In self-regulated learning, the goals involve learning and with regulatory processes we refer to those processes which learners use to adapt to the challenges in a learning situation in order to progress their learning goals. Combining different data channels (e.g., videos and physiological data) still need researcher's interpretation and coding, but the SLAM-KIT is a first attempt to make the unfolding process data visual and, thus, help learning scientist to understand the complex set of data. Learning processes that could be studied based only on subjective data can be verified objectively and triangulated with different types of data. The use of self-reported data alone is not advocated in the literature because such self-reported data often do not match with what is actually happening during learning processes (Winne, 2004; Zimmerman, 2008). Typically, the annotation schemas (e.g., video and verbal 
protocols) used by researchers to analyse data are not only time-consuming and expensive to create, but also tied to the specific content and situational context (Noroozi et al., 2013). Furthermore, such codings are typically subject to interpretation and should therefore be treated cautiously especially when generalizing the findings to other collaborative learning settings.

Advanced technologies can provide rich data to investigate a range of cognitive and non-cognitive learning processes during collaboration. The transition into new digital workflows and protocols along with subsequent automated analysis and use of reliable sensor data can speed up investigations and produce big data about complex collaborative learning processes. Physiological data is a reliable source of data that can be used to cover constraints of such self-reported and observational data. We discussed each of these physiological data (e.g., EDA, Eye-movement, facial expression, heart rate) in relation to the collaborative learning regulatory processes. However, the challenge is how to decide upon and matching the granularity of the data from each source. So, assigning and enforcing a synchronized level of granularity could be difficult. Some data is fine grained, for example, changes in the heart rate can occur rapidly and be short in duration. Other types of data, such as facial expressions, are less fine-grained (i.e., fewer categories and of comparatively longer duration). A final limitation is that one datum (i.e., a change in the heart rate) could be due to multiple causes requiring painstaking - sometimes subjective - human interpretation of contextual data. Such physiological and technology-assisted data collection produces a significant amount of data (i.e., big data), which increases the number of data challenges researchers must overcome in order to handle, analyse, and understand the data gathered (D'Mello, Duckworth, \& Dieterle, 2017).

Our aim, presented in this paper, is to simplify the use and analysis of rich multimodal data by developing an integrated analysis tool, SLAM-KIT, which enables the user to approach interaction situations using a wealth of biometric information captured using unobtrusive sensors and cameras. The tool merges the diverse data sources together and provides a unified navigable view of the entire interaction situation. The SLAM-KIT is designed to provide the learning science community a unified tool to study synchronized physiological signals of the participants in a learning session aligned with the recorded video and preprocessed annotations. SLAM-KIT also enables users to choose and execute simple analysis algorithms to identify and measure phenomena relevant in their investigation. The ultimate goal is to provide learning scientists with easy access to all of the physiological information relevant in the SRL, SSRL and CoRL approaches to analysing the learning situations.

Currently, the SLAM-KIT is used by researchers, but we are intensively working on the tool to target teachers and learners as well. Wider availability and further field studies are seen as a future step. For example, the SLAM-KIT tool could provide learners with mental and metacognitive learning 
processes in terms of visualisation. With such information and awareness of their learning process, learners can become more independent and thus better regulate their learning activities (Wang \& Jacobson, 2011; Wang et al., 2011). SLAM-KIT is different from other commercial sector products such as Tobii. The Tobii products are primarily designed for eye tracking solutions, however SLAMKIT is a piece of software customized for visualization and analysis of multimodal biosignals recorded for a group of subjects simultaneously with their video.

Machine learning or automatic classification of signals are not utilized in the tool presently. Instead, visualisation and signal processing methods are used merely for studying and understanding the relationship between self-regulated learning and the bio-signals in a group setting. Our future work includes development of signal analysis functions that could be used to predict/classify the cognitive processes and anticipate the labeled annotations.

At this point, we do not have any formal tests of perceived usefulness, ease of use, and intention to use of the tool, but we collected informal feedback from the SLAM users and how it was taken into account while developing the tool. We have intended users on the list of authors, so at this point we are talking about a prototype to investigate whether bio-signals make sense in this context. The team of SLAM researchers are currently busy with formal testing of the tool which is also valuable for commercialisation of the tool.

Triangulation of data for regulated learning theory is still in its infancy especially when it comes to collaborative learning settings. The learning sciences lack a comprehensive yet systematic literature review of the current multimodal data that can be used for collaborative learning. Therefore, it is advised to conduct a systematic review study to see what multimodal data is available in the field and how they can be used to facilitate learning regulation in collaborate settings. We propose that researchers continue to integrate interdisciplinary methodologies to capture regulated learning using trace methodologies, such as log-file data, eye-movement data, physiological measures, video data, and self-report measures of learning processes. These methodologies are important to extend the methodological paradigm in the area of SRL, CoRL and SSRL with new, interdisciplinary work using innovative tools and techniques from educational data mining, machine learning, and affective computing (Baker \& Siemens, 2014)

\section{References}

Antonietti, A., Colombo, B., \& Di Nuzzo, C. (2014). Metacognition in self-regulated multimedia learning: integrating behavioural, psychophysiological and introspective measures. Learning, Media and Technology, 40(2), 1-23. 
Azevedo, R. (2015). Defining and measuring engagement and learning in science: Conceptual, theoretical, methodological, and analytical issues. Educational Psychologist, 50(1), 84-94.

Baker, R., \& Siemens, G. (2014). Educational data mining and learning analytics. In K. Sawyer(Ed.), Cambridge handbook of the learning sciences (2nd ed., pp. 253-274). Cambridge, MA: Cambridge University Press.

Bannert, M., Reimann, P., \& Sonnenberg, C. (2014). Process mining techniques for analysing patterns and strategies in students' self-regulated learning. Metacognition and Learning, 9(2), $161-185$.

Basol, G., \& Balgalmis, E. (2016). A multivariate investigation of gender differences in the number of online tests received-checking for perceived self-regulation. Computers in Human Behavior, 58, 388-397.

Blikstein, P., Gomes, J.S., Akiba, H.T., \& Schneider, B. (2016). The effect of highly scaffolded versus general instruction on students' exploratory behavior and arousal. Technology, Knowledge and Learning, 22(1), 105-128.

Chanel, G., \& Mühl, C. (2015). Connecting brains and bodies: Applying physiological computing to support social interaction. Interacting with Computers, 27(5), 534-550.

Cranford, K.N., Tiettmeyer, J.M., Chuprinko, B.C., Jordan, S., \& Grove, N.P. (2014). Measuring load on working memory: The use of heart rate as a means of measuring chemistry students' cognitive load. Journal of Chemical Education, 91(5), 641-647.

D'Mello, S. (2013). A selective meta-analysis on the relative incidence of discrete affective states during learning with technology. Journal of Educational Psychology, 105(4), 1082-1099.

D'Mello, S.K., Duckworth, A., \& Dieterle, E. (2017). Advanced, Analytic, Automated (AAA) Measurement of Engagement during Learning. Educational Psychologist, 57(2), 104-123.

Fairclough, S.H., Venables, L., \& Tattersall, A. (2005). The influence of task demand and learning on the psychophysiological response. International Journal of Psychophysiology, 56(2), 171184.

Garbarino, M., Lai, M., Tognetti, S., Picard, R., \& Bender, D. (2014). Empatica E3 - A wearable wireless multi-sensor device for real-time computerized biofeedback and data acquisition. Proceedings of the 4th International Conference on Wireless Mobile Communication and Healthcare - "Transforming Healthcare through Innovations in Mobile and Wireless Technologies," 39-42. http://doi.org/10.4108/icst.mobihealth.2014.257418. 
Gašević, D., Dawson, S., \& Siemens, G. (2015). Let's not forget: Learning analytics are about learning. TechTrends, 59(1), 64-71.

Greller, W., \& Drachsler, H. (2012). Translating learning into numbers: Toward a generic framework for learning analytics. In G. Siemens (Ed.), Educational Technology and Society, Special Issue on Learning Analytics (pp. 42-57). http://doi.org/10.1145/2330601.2330634.

Hadwin, A.F., Järvelä, S., \& Miller, M. (2017). Self-regulation, co-regulation and shared regulation in collaborative learning environments (pp. 83-106). In D. Schunk, \& J. Greene, (Eds.). Handbook of Self-Regulation of Learning and Performance, 2nd Ed. NY: Routledge.

Haapalainen, E., Kim, S., Forlizzi, J.F., \& Dey, A.K. (2010). Psycho-physiological measures for assessing cognitive load. Proceedings of the 12th ACM International Conference on Ubiquitous Computing, 301-310.

Harley, J.M., Bouchet, F., Hussain, M.S., Azevedo, R., \& Calvo, R. (2015). A multicomponential analysis of emotions during complex learning with an intelligent multiagent system. Computers in Human Behavior, 48, 615-625.

Henriques, R., Paiva, A., \& Antunes, C. (2013). On the need of new methods to mine electrodermal activity in emotion-centered studies. In L. Cao, Y. Zeng, A.L. Symeonidis, V.I. Gorodetsky, P.S. Yu, \& M.P. Singh (Eds.), Agents and Data Mining Interaction (Vol. 7607, pp. 203-215). Berlin: Springer.

Jarodzka, H., Holmqvist, K., \& Gruber, H. (2017). Eye tracking in educational science: Theoretical frameworks and research agendas. Journal of Eye Movement Research, 10(1), 1-18.

Järvelä, S., \& Hadwin, A.F. (2013). New frontiers: Regulating learning in CSCL. Educational Psychologist, 48(1), 25-39.

Järvelä, S., Järvenoja, H., Malmberg, J., Isohätälä, J., \& Sobocinski, M. (2016). How do types of interaction and phases of self-regulated learning set a stage for collaborative engagement? Learning and Instruction, 43(1), 39-51.

Kreibig, S.D., Gendolla, G.H.E., \& Scherer, K.R. (2012). Goal relevance and goal conduciveness appraisals lead to differential autonomic reactivity in emotional responding to performance feedback. Biological Psychology, 91(3), 365-375.

Leite, I., Henriques, R., Martinho, C., \& Paiva, A. (2013). Sensors in the wild: Exploring electrodermal activity in child-robot interaction. Human-Robot Interaction (HRI) Proceedings 
of the 8th ACM/IEEE International Conference on Human-Robot Interaction (pp. 41-48). IEEE Press Piscataway, NJ, USA.

Li, X., Hong, X., Moilanen, A., Huang, X., Pfister, T., Zhao, G., \& Pietikainen, M. (2017). Towards reading hidden emotions: a comparative study of spontaneous micro-expression spotting and recognition methods. IEEE Transactions on Affective Computing, 99. http://dx.doi.org/10.1109/TAFFC.2017.2667642.

Malmberg, J., Järvelä, S., \& Järvenoja, H., (2017). Capturing temporal and sequential patterns of self, co- and socially shared regulation in the context of collaborative learning. Contemporary Educational Psychology, 49(1). 160-174.

Mikroyannidis, A., Okada, A., Scott, P., Rusman, E., Specht, M., Stefanov, K., ... Chaimala, F. (2013). WeSPOT: A personal and social approach to inquiry-based learning. Journal of Universal Computer Science, 19(14), 2093-2111.

Miller, M., \& Hadwin, A. (2015). Scripting and awareness tools for regulating collaborative learning: Changing the landscape of support in CSCL. Computers in Human Behavior, 52(1), 573-588.

Molenaar, I., \& Chiu, M.M. (2014). Dissecting sequences of regulation and cognition: Statistical discourse analysis of primary school children's collaborative learning. Metacognition and Learning, 9(2), 137-160. http://doi.org/10.1007/s11409-013-9105-8.

Mønster, D., Håkonsson, D.D., Eskildsen, J.K., \& Wallot, S. (2016). Physiological evidence of interpersonal dynamics in a cooperative production task. Physiology \& Behavior, 156(1), 2434.

Nistor, N., \& Hernández-Garcíacc, A. (2018). What types of data are used in learning analytics? An overview of six cases. Computers in Human Behavior, 89, 335-338.

Noroozi, O., Kirschner, P., Biemans, H.J.A., \& Mulder, M. (2018). Promoting argumentation competence: Extending from first- to second-order scaffolding through adaptive fading. Educational Psychology Review, 30(1), 153-176.

Noroozi, O., Teasley, S.D., Biemans, H.J.A., Weinberger, A., \& Mulder, M. (2013). Facilitating learning in multidisciplinary groups with transactive CSCL scripts. International Journal of Computer-Supported Collaborative Learning, 8(2), 189-223.

Noroozi, O., Weinberger, A., Biemans, H.J.A., Mulder, M., \& Chizari, M. (2012). Argumentationbased computer supported collaborative learning (ABCSCL). A systematic review and synthesis of fifteen years of research. Educational Research Review, 7(2), 79-106. 
Pecchinenda, A., \& Smith, C.A. (1996). The affective significance of skin conductance activity during a difficult problem-solving task. Cognition \& Emotion, 10(5), 481-504.

Pijeira-Díaz, H.J., Drachsler, H., Järvelä, S., \& Kirschner, P.A. (2016). Investigating collaborative learning success with physiological coupling indices based on electrodermal activity. Proceedings of the Sixth International Conference on Learning Analytics and Knowledge. ACM. Edinburgh, United Kingdom. April 25-29, 2016.

Reimann, P., Markauskaite, L., \& Bannert, M. (2014). E-research and learning theory: What do sequence and process mining methods contribute? British Journal of Educational Technology, $45(3), 528-540$.

Roll, I., \& Winne, P.H. (2015). Understanding, evaluating, and supporting self-regulated learning using learning analytics. Journal of Learning Analytics, 2(1), 7-12.

Tomaka, J., Blascovich, J., Kelsey, R.M., \& Leitten, C.L. (1993). Subjective, physiological, and behavioral effects of threat and challenge appraisal. Journal of Personality and Social Psychology, 65(2), 248-260.

Verbert, K., Duval, E., Klerkx, J., Govaerts, S., \& Santos, J.L. (2013). Learning analytics dashboard applications. American Behavioral Scientist, 57(10), 1500-1509.

Viberg, O., Hatakka, M., Bälter, O., \& Mavroudi, A. (2018). The current landscape of learning analytics in higher education. Computers in Human Behavior, 89, 98-110.

Wallot, S., Roepstorff, A., \& Mønster, D. (2016). Multidimensional Recurrence Quantification Analysis (MdRQA) for the analysis of multidimensional time-series: A software implementation in MATLAB and its application to group-level data in joint action. Frontiers in Psychology, 7, 1835. http://doi.org/10.3389/fpsyg.2016.01835.

Wang, M., Peng, J., Cheng, B., Zhou, H., \& Liu, J. (2011). Knowledge Visualization for SelfRegulated Learning. Educational Technology \& Society, 14(3), 28-42.

Wang, M., \& Jacobson, M.J. (2011). Knowledge Visualization for Learning and Knowledge Management. Educational Technology \& Society, 14(3), 1-3.

Winne, P.H. (2004). Comments on motivation in real-life, dynamic, and interactive learning environments. European Psychologist, 9(4), 257-263. 
Winne, P.H. (2017). Cognition and metacognition in self-regulated learning. In D. Schunk \& J. Greene (Eds.). Handbook of self-regulation of learning and performance. (2nd Ed.). New York, NY: Routledge.

Winne, P.H. (2010). Improving measurements of self-regulated learning. Educational Psychologist, 45(4), 267-276.

Winne, P.H., \& Hadwin, A.F. (1998). Studying as self-regulated engagement in learning. In D. Hacker, J. Dunlosky, \& A. Graesser (Eds.), Metacognition in Educational Theory and Practice (pp. 277-304). Hillsdale, NJ: Lawrence Erlbaum.

Wise, F.A., \& Hsiao, T.Y. (in press). Self-regulation in online discussions: Aligning data streams to investigate relationships between speaking, listening, and task conditions. Computers in Human Behavior. https://doi.org/10.1016/j.chb.2018.01.034.

Worsley, M., \& Blikstein, P. (2015). Leveraging multimodal learning analytics to differentiate student learning strategies. In Proceedings of the Fifth International Conference on Learning Analytics and Knowledge - LAK'15 (pp. 360-367). New York, USA: ACM Press.

Zhang, X., Meng, Y., Ordóñez de Pablos, P., Sun, Y. (in press). Learning analytics in collaborative learning supported by slack: from the perspective of engagement. Computers in Human Behavior. https://doi.org/10.1016/j.chb.2017.08.012.

Zimmerman, B.J. (2008). Investigating self-regulation and motivation: Historical background, methodological developments, and future prospects. American Educational Research Journal, 45(1), 166-183.

Zimmerman, B.J., \& Schunk, D.H. (2011). Self-regulated learning and performance: An Introduction and an overview. In B.J. Zimmerman \& D.H. Schunk (Eds.), Handbook of Self-Regulation of Learning and Performance (pp. 1-12). New York: Routledge. 